\section{Paediatric vs adult retinal detachment}

S Rumelt ${ }^{1,2}$, L Sarrazin² ${ }^{2}$ E Averbukh², M Halpert ${ }^{2}$ and I Hemo ${ }^{2}$

\begin{abstract}
Objective To evaluate the causes, incidences, characteristics, and treatment outcomes of paediatric $v s$ adult retinal detachment. Patients and methods One hundred and sixty (136 patients) out of 2408 consecutive retinal detachments $(6.6 \%)$ at our facility occurred in children under the age of 18 years. Of them, 144 eyes $(90 \%)$ of $127(93 \%)$ children were treated and compared with a sample of 56 consecutive retinal detachments in $\mathbf{5 0}$ adults (over the age of 18 years). The parameters for comparison included cause, type of retinal detachment, its extent, macular involvement, number of tears, number and types of surgery, and the anatomic
\end{abstract} and functional surgical outcome.

Results Statistically significant differences were found in the type of retinal detachment. Rhegmatogenous RD was less common $(P=0.004)$, and exudative $R D$ was more common $(P=0.021)$ in the paediatric group. Ocular trauma and ocular syndromes were more common in the paediatric group $(P<0.001)$, while myopia, posterior vitreous detachment, and retinal detachment following cataract surgery were less common in this group compared with adults $(P<0.001,<0.001$, and 0.001 , respectively). Ocular pathologies associated with retinal detachment were more common in the paediatric group $(P<0.001)$. Initial and last visual acuity of $>20 / 400$, last visual acuity of $>20 / 40$, and retinal complete reattachment were higher in adults $(P<0.001)$. Conclusions The type of retinal detachment, causes and outcomes were statistically different between paediatric and adult cases. The less successful functional and anatomical outcomes of retinal detachment surgery in children may reflect the different aetiologies and indicate the need for aetiology-specific treatment strategies according to each aetiology.

Eye (2007) 21, 1473-1478; doi:10.1038/sj.eye.6702511; published online 28 July 2006

Keywords: Paediatric retinal detachment; adult retinal detachment; children surgical treatment; anatothical outcome; visual outcome

\section{Introduction}

Rhegmatogenous retinal detachment is the common form of retinal detachment and occurs in approximately 12.4 persons per 100000 population. ${ }^{1}$ In children under 18 years of age, it accounts for $3.2-5.6 \%$ of all the incidents of retinal detachment. The major cause for retinal detachment in the young is ocular trauma followed by myopia. ${ }^{2}$ Exudative retinal detachment is rarer and is caused usually secondary to ocular syndromes such as Coats' disease. Other rare form of detachment is tractional occurring with other conditions such as ocular trauma. ${ }^{3}$ Several papers have addressed retinal detachment in children and adolescents, $^{1-9}$ but we are unaware of any comparative study between paediatric and adult retinal detachment and could not find such a study using data search on Medline ${ }^{\circledR}$. Although paediatric and adult retinal detachments involve two heterogeneous groups, a comparative study between paediatric and adult population may demonstrate whether the preoperative aetiologies, characteristics and outcomes are similar and if not, whether they can they be associated with each other.

Therefore, we conducted this study to elucidate the differences in aetiology, type, and other characteristics of retinal detachment between the groups and the functional and visual surgical outcomes of these two groups.

\section{Patients and methods}

We reviewed the charts of all patients that were diagnosed with retinal detachment at Hadassah University Hospital between 1980 and 2000. One hundred and sixty (136 Caucasian patients) out of 2408 consecutive retinal detachments $(6.6 \%)$ that were treated occurred in children under the age of 18 years. Of them, 144 eyes $(90 \%)$ of $127(93 \%)$ children were treated and compared with an arbitrary sample of 56 retinal detachments in 50 Caucasian adults (over the age of 18 years). Excluded were 13 retinal detachments due to retinopathy of prematurity
${ }^{1}$ Department of Ophthalmology, Western Galilee - Nahariya Medical Center, Nahariya, Israel

${ }^{2}$ Hadassah University Hospital, Jerusalem, Israel

Correspondence: S Rumelt, Department of Ophthalmology, Western Galilee - Nahariya Medical Center, PO Box 21, 22100 Nahariya, Israel Tel: + 97249107635 ; Fax: + 97249107611. E-mail: rumelt@ naharia.health.gov.il

Received: 11 March 2005 Accepted in revised form: 6 June 2006 Published online: 28 July 2006

Presented in part at the 107th American Academy of Ophthalmology Annual Meeting, Anaheim, CA, November, 2003, The Red Sea International Update conference in Ophthalmology, Eilat, Israel, June 2004 and 30th International Congress of Ophthalmology, Sao Paolo, Brazil, February 2006.

The authors do not have financial or propriety interest in any of the products or procedures mentioned in this paper 
and 10 retinal detachments nonamenable for treatment or without detailed record. The treated-children age range was 3 months to 18 years (mean $\pm \mathrm{SD}=10.8 \pm 5.08$ years) and the adult age range was $32-85$ years (mean $\pm \mathrm{SD}=58.4 \pm 13.5$ years). Comparison was conducted for the cause of retinal detachment, type of retinal detachment (rhegmatogenous, exudative, or traction), its extent, macular involvement, number of tears, associated ocular pathologies such as colobomata, number and types of surgeries, and the anatomic and functional surgical outcome. All surgeries were performed at one medical center by the same team.

Surgery was performed as previously detailed and according to established criteria for both groups. ${ }^{10}$ Basically, buckle procedure with or without external drainage was performed in all cases of idiopathic rhegmatogenous retinal detachment except when amenable to pneumatic retinopexy. Pars plana vitrectomy was performed if redetachment occurred or as a primary procedure in cases of risk for proliferative vitreoretinopathy (vitreous cells). Exudative retinal detachment was treated by systemic corticosteroids, argon laser and cryotherapy, pars plana vitrectomy, and/ or buckling with external drainage according to the extent and the aetiology. In our series, exudative retinal detachments were caused mostly by Coats' disease. Small exudative detachments may absorb with systemic corticosteroids, argon laser and cryotherapy that cause destruction or regression of vascular leaking lesions and may stimulate fluid absorption by retinal pigment epithelial cells. ${ }^{10,11}$ In cases of extensive exudative detachments, additional buckling and vitrectomy with drainage allow more fluid to absorb and may alleviate or prevent traction elements. ${ }^{12}$ Traumatic retinal detachments were treated by three-port pars plana vitrectomy with removal of the cortical and incarcerated vitreous, endolaser, and scleral encircling buckling. Lensectomy was performed for all cases with traumatic cataract and anterior proliferative vitreoretinopathy. Membrane peeling was performed in cases of epiretinal membranes causing retinal traction or associated with visual acuity of less than $20 / 80$. In cases of proliferative vitreoretinopathy, giant retinal tears and reoperations, silicone oil was employed and was removed in cases of complications or after stable reattachment for 6 months. Intraocular foreign bodies from penetration were removed with intraocular forceps. In children with ocular trauma, surgery was performed within 3-7 days from the diagnosis to allow vitreous liquefaction and before the development of proliferative vitreoretinopathy. In children with retinal detachment from causes other than trauma and in adults, surgery was performed within 1 or 2 days. Children under the age of 10 years were treated with occlusive therapy for amblyopia immediately after surgery. Follow-up period extended until inoperable redetachment or the end of the study (at least a year from presentation). Patients were followed each day until reattachment, 1, 3, 6, and 12 months after surgery and then annually.

The follow-up period for adult retinal detachment was between 1 month and 18 years (average 3.23 years) and for paediatric retinal detachment between 1 month and 16 years (average 3.69 years). The follow-up evaluation included visual acuity, intraocular pressure, and complete eye examination including funduscopy after pupil dilation. Anatomical success was defined as complete retinal reattachment. Functional outcome was assessed by visual acuity using Snellen chart or its equivalent (forced preferential looking) in nonverbal children, under constant illumination.

Each of the confluent covariates except initial visual acuity was assessed with Wilcoxon rank sum test. Each of the categorical covariates was individually assessed with $\chi^{2}$-test. Fisher exact test was employed for samples with expectancy of less than 5 and a $P<0.05$ was considered statistically significant. Logistic regression model or Bonferoni correction was not employed due to the limited number of retinal detachments. The sample size had $80 \%$ power to detect $20 \%$ differences between children and adults with retinal detachment in two-tail test and a type I error of 5\%. IRB or institutional approval for this study was not required.

\section{Results}

\section{Retinal detachment types, causes, and incidences}

Statistically significant differences were found in the type of retinal detachment. Rhegmatogenous retinal detachment was less common $(P=0.004)$, and exudative retinal detachment was more common $(P=0.021)$ in the paediatric group (Table 1). The most common aetiologies for rhegmatogenous and exudative retinal detachments in children were trauma (in 61 out of 126 eyes, 48\%) and Coats' disease (in five out of eight eyes, 63\%), respectively. In adults, the most common attributing factor for rhegmatogenous detachment was myopia (53\%) followed by idiopathic posterior vitreous detachment $(16 \%)$. No statistically significant differences were found in the occurrence of traction retinal detachment and combined rhegmatogenous and traction retinal detachment between the two groups, but only a limited number of patients had these types of detachment. Differences were also insignificant in the occurrence of retinal dialysis and marginal in giant retinal tears between the groups.

Ocular trauma and ocular syndromes were more common in the paediatric age group $(P<0.001)$, while 
Table 1 The parameters of paediatric and adult retinal detachment and their statistical significance

\begin{tabular}{|c|c|c|c|c|}
\hline Parameter & Children $(\mathrm{n}=127)$ eyes $(\mathrm{n}=144)$ & Adults $(\mathrm{n}=50)$ eyes $(\mathrm{n}=56)$ & P-value & Statistical test \\
\hline \multicolumn{5}{|l|}{ Gender } \\
\hline Male & $93(73 \%)$ & $32(64 \%)$ & 0.225 & $\chi^{2}$ \\
\hline Female & $34(27 \%)$ & $18(36 \%)$ & 0.225 & $\chi^{2}$ \\
\hline Bilateral & $20(16 \%)$ & $6(12 \%)$ & 0.526 & $\chi^{2}$ \\
\hline \multicolumn{5}{|l|}{ Type of $R D$} \\
\hline Rhegmatogenous & $115(80 \%)$ & $54(96 \%)$ & 0.004 & $\chi^{2}$ \\
\hline Exudative & $13(9 \%)$ & $0(0 \%)$ & 0.021 & Fisher exact \\
\hline Traction & $15(10 \%)$ & $2(3.6 \%)$ & 0.161 & Fisher exact \\
\hline Combined & $1(0.7 \%)$ & $0(0 \%)$ & 1.000 & Fisher exact \\
\hline \multicolumn{5}{|l|}{ Other characteristics } \\
\hline Giant retinal tear & $19(13 \%)$ & $2(3.6 \%)$ & 0.046 & $\chi^{2}$ \\
\hline Dialysis & $2(1.4 \%)$ & $0(0 \%)$ & 1.000 & Fisher exact \\
\hline \multicolumn{5}{|l|}{ Causes/risk factors } \\
\hline Ocular trauma & $61(42 \%)$ & $3(5 \%)$ & $<0.001$ & $\chi^{2}$ \\
\hline High myopia (>6.00D) & $16(11 \%)$ & $17(30 \%)$ & 0.001 & $\chi^{2}$ \\
\hline Myopia $(<6.00 \mathrm{D})$ & $5(3 \%)$ & $11(20 \%)$ & $<0.001$ & Fisher exact \\
\hline Idiopathic PVD & $1(0.7 \%)$ & $9(16 \%)$ & $<0.001$ & Fisher exact \\
\hline Ocular syndromes & $52(36 \%)$ & $0(0 \%)$ & $<0.001$ & Fisher exact \\
\hline After cataract surgery & $14(10 \%)$ & $15(27 \%)$ & 0.001 & $\chi^{2}$ \\
\hline Others/idiopathic & $31(21 \%)$ & $1(2 \%)$ & $<0.001$ & Fisher exact \\
\hline Initial visual acuity $>20 / 400$ & $43(30 \%)$ & $32(57 \%)$ & $<0.001$ & $\chi^{2}$ \\
\hline Macular involvement & $111(77 \%)$ & $25(45 \%)$ & $<0.001$ & $\chi^{2}$ \\
\hline Associated ocular pathologies & $98(62 \%)$ & $9(16 \%)$ & $<0.001$ & $\chi^{2}$ \\
\hline Postoperative complications & $83(58 \%)$ & $19(34 \%)$ & 0.003 & $\chi^{2}$ \\
\hline Anatomic success & $75(52 \%)$ & $50(89 \%)$ & $<0.001$ & $\chi^{2}$ \\
\hline Final visual acuity $>20 / 400$ & $54(37 \%)$ & $46(82 \%)$ & $<0.001$ & $\chi^{2}$ \\
\hline Final visual acuity $>20 / 40$ & $18(12 \%)$ & $26(46 \%)$ & $<0.001$ & $\chi^{2}$ \\
\hline
\end{tabular}

myopia, posterior vitreous detachment, and retinal detachment following cataract surgery were less common in this group compared with adults $(P<0.001$, $<0.001$, and 0.001 , respectively). Lattice degeneration was found in $4.9 \%$ of the children eyes and $1.5 \%$ of the adults' eyes $(P=0.447)$. Ocular pathologies (eg, lens or uveal colobomata, primary hyperplastic vitreous, and vitreoretinopathy) associated with retinal detachment were more common in the paediatric group $(P<0.001)$.

\section{Interventions for retinal detachment}

In the paediatric group, $109(67 \%)$ eyes underwent a single intervention, 31 (19\%) underwent two surgeries, $12(8 \%)$ underwent three surgeries, 4 (3\%) underwent four surgeries, and 4 (3\%) additional eyes underwent five, six, seven, or eight surgeries. In the adult group, 34 (61\%) underwent a single surgery, 10 (18\%) underwent two surgeries, 8 (14\%) underwent three surgeries, and 4 (7\%) additional eyes underwent four, five, six, or seven surgeries. There were no significant statistical differences between the groups in the number of interventions.

\section{Outcomes and complications of retinal detachment}

Statistically significant differences were found in the functional and anatomical outcomes between paediatric and adult retinal detachment. Initial and last visual acuity of $>20 / 400$, last visual acuity of $>20 / 40$ and retinal reattachment were less frequent in children $(P<0.001)$. Anatomical success was obtained more commonly and visual acuity of 20/200 was marginally better in rhegmatogenous compared with exudative retinal detachment $(P<0.001$ and $P=0.057$, respectively). Anatomic success was achieved in $14(21 \%)$ of the eyes that had retinal redetachment in the paediatric group and in $13(65 \%)$ eyes that had retinal redetachment in the adult group $(P<0.001)$. Some eyes had more than one episode of retinal redetachment.

Table 2 shows the number and percentage of different complications in the paediatric $v s$ the adult retinal detachment groups. A total of $83(58 \%)$ out of the 144 eyes in the paediatric group and 19 (34\%) out of the 56 eyes in the adult population had one complication or more $(P=0.003)$. In all, $34(21 \%)$ eyes in the paediatric 
Table 2 The individual intra and postoperative complications in paediatric and adult retinal detachment. Some of the eyes had more than one complication

\begin{tabular}{|c|c|c|c|c|}
\hline Complications & Children eyes $(\mathrm{n}=144)$ & Adult eyes $(\mathrm{n}=56)$ & P-value & Statistical test \\
\hline Overall no. of eyes with complications & $83(58 \%)$ & $19(34 \%)$ & 0.003 & $\chi^{2}$ \\
\hline Retinal redetachment & $68(47 \%)$ & $20(36 \%)$ & 0.141 & $\chi^{2}$ \\
\hline Retinal non-attachment & $10(7 \%)$ & $3(5 \%)$ & 1.000 & Fisher exact \\
\hline New retinal tears & $4(3 \%)$ & $2(4 \%)$ & 0.673 & Fisher exact \\
\hline Giant retinal tear & $2(1 \%)$ & $0(0 \%)$ & 1.000 & Fisher exact \\
\hline Retinal dialysis & $1(0.7 \%)$ & $0(0 \%)$ & 1.000 & Fisher exact \\
\hline Macular hole & $2(1 \%)$ & $1(2 \%)$ & 1.000 & Fisher exact \\
\hline Intraoperative suprachoroidal haemorrhage & $1(0.7 \%)$ & $0(0 \%)$ & 1.000 & Fisher exact \\
\hline Subretinal fibrosis & $5(3 \%)$ & $2(4 \%)$ & 1.000 & Fisher exact \\
\hline Macular pucker & $4(3 \%)$ & $3(5 \%)$ & 0.402 & Fisher exact \\
\hline Cystoid macular edema & $0(0 \%)$ & $1(2 \%)$ & 0.280 & Fisher exact \\
\hline Anterior ischemic optic neuropathy & $0(0 \%)$ & $1(2 \%)$ & 0.280 & Fisher exact \\
\hline Optic nerve atrophy & $1(0.7 \%)$ & $0(0 \%)$ & 1.000 & Fisher exact \\
\hline Proliferative vitreoretinopathy & $18(12 \%)$ & $3(5 \%)$ & 0.199 & $\chi^{2}$ \\
\hline Cataract & $9(6 \%)$ & $5(9 \%)$ & 0.541 & Fisher exact \\
\hline Secondary cataract & $4(3 \%)$ & $0(0 \%)$ & 0.578 & Fisher exact \\
\hline Secondary glaucoma & $6(4 \%)$ & $2(4 \%)$ & 1.000 & Fisher exact \\
\hline Cyclitic membrane & $1(0.7 \%)$ & $0(0 \%)$ & 1.000 & Fisher exact \\
\hline Anterior chamber silicone oil emulsification & $6(4 \%)$ & $2(4 \%)$ & 1.000 & Fisher exact \\
\hline Band keratopathy & $13(9 \%)$ & $1(2 \%)$ & 0.111 & Fisher exact \\
\hline Corneal erosion/ulcer & $2(1.4 \%)$ & $0(0 \%)$ & 1.000 & Fisher exact \\
\hline Hemophthalmos & $3(2 \%)$ & $0(0 \%)$ & 0.561 & Fisher exact \\
\hline endophthalmitis & $0(0 \%)$ & $1(2 \%)$ & 0.280 & Fisher exact \\
\hline Sympathetic ophthalmia & $1(0.7 \%)$ & $0(0 \%)$ & 1.000 & Fisher exact \\
\hline Band protrusion & $1(0.7 \%)$ & $0(\%)$ & 1.000 & Fisher exact \\
\hline Band-induced myopia & $1(0.7 \%)$ & $0(0 \%)$ & 1.000 & Fisher exact \\
\hline Phtisis bulbi & $10(7 \%)$ & $2(4 \%)$ & 0.516 & Fisher exact \\
\hline Esotropia & $4(3 \%)$ & $0(0 \%)$ & 0.578 & Fisher exact \\
\hline Exotropia & $1(0.7 \%)$ & $0(0 \%)$ & 1.000 & Fisher exact \\
\hline Intractable ocular pain & $2(1.4 \%)$ & $0(0 \%)$ & 1.000 & Fisher exact \\
\hline Wound dehiscence & $1(0.7 \%)$ & $0(0 \%)$ & 1.000 & Fisher exact \\
\hline
\end{tabular}

group and $13(23 \%)$ eyes in the adult group had more than a single complication $(P=1.000)$. No statistically significant differences were found in each of the complications, including postoperative proliferative vitreoretinopathy, between the groups.

\section{Discussion}

\section{Retinal detachment types, causes, and incidences}

Retinal detachment was infrequent in children compared with adults even in regard to their proportion in the population. The incidence was similar to that reported in the literature. ${ }^{2}$ The low incidence of detachment in children may be attributed to differences in the aetiology of the detachment. In children, ocular syndromes and pathologies were considered infrequent compared with the acquired ocular pathologies such as posterior vitreous detachment in adults.

Retinal detachment occurred in a similar incidence in males and in females in both paediatric and adult groups. The incidence was similar to some reports in the literature ${ }^{5,6,9}$ although traumatic retinal detachment was higher in males than in females in both groups (data not shown) as found also by others., ${ }^{4,12-15}$

In our study, rhegmatogenous retinal detachment was statistically less common and exudative retinal detachment was statistically more common in the paediatric group than in adults. The differences reflected the different aetiologies for retinal detachment in children and adults. While myopia and posterior vitreous detachment were major risk factors for retinal detachment in adults, ocular syndromes such Coats' disease resulted in exudative type of retinal detachment. Although exudative retinal detachment was more common in children than in adults, it was still less common in this group than rhegmatogenous type, since associated aetiologies such as ocular trauma were statistically more common than the pathologies resulting in exudative retinal detachments. Indeed, ocular trauma was a common cause for rhegmatogenous retinal detachment in children in other studies and occurred in 
25-61\%., ${ }^{73,16-18}$ Ocular and systemic syndromes were found in higher proportion in our paediatric population compared with other studies., ${ }^{7,16}$ This probably reflects our larger sample. Comparative data are not available in the literature, but in other noncomparative studies, retinal detachment in congenital myopia was associated with giant retinal tears. ${ }^{8,19,20}$ In our population, giant retinal tear was not a common finding, probably because most of the patients developed myopia during childhood and did not include only the congenital ones. Ora dialysis was found commonly in paediatric age group $(20-42 \%),{ }^{13,20,21}$ but not in our study. The occurrence of lattice degeneration was similar to another study in which it was $5.8 \%$ of the paediatric retinal detachments. ${ }^{7}$ This type of degeneration is found usually in older children of 15-19 years that are more similar to adults ${ }^{20}$ and in up to $61 \%$ of the 'idiopathic' rhematogenous retinal detachments. ${ }^{22}$

We found that ocular pathologies other than retinal detachment were more statistically common in the paediatric group and the aetiologies were different. To our best knowledge, comparison between paediatric and adult populations has never been made but one study found that in younger children there are frequent ocular pathologies other than retinal detachment compared with older children..$^{20}$ Some of these aetiologies are associated with early onset and therefore do not manifest in adulthood. Posterior vitreous detachment on the contrary is common in adults, while almost never encountered in children due to tight retinal-vitreous adherence. ${ }^{23}$ Posterior vitreous detachment occurs frequently after cataract extraction in adults and predisposes for retinal detachment. ${ }^{24}$ In children, posterior vitreous and retinal detachments may be encountered following extraction of traumatic cataracts and not spontaneously.

\section{Outcomes and complications of retinal detachment}

The different causes for retinal detachment, associated ocular pathologies, and occurrence of postoperative complications may be attributed to the worse anatomical and functional outcomes in children rather than the number of interventions. We found that the number of interventions was similar between the groups, although it might be expected that in children with poorer anatomical and functional outcomes, the number of surgical interventions would be higher. We found that in eyes complicated after surgery, there was a tendency for more than one complication and adults had a higher success rate or retinal reattachment after redetachment. Proliferative vitreoretinopathy was comparable in the paediatric and the adult group. ${ }^{25-30}$ In our study, it was less common than previously reported for children and at a rate similar to that of adults. This may be related to the difficulties in precise examination of the retinal periphery in children as well to the inherent difficulties associated with retrospective study. Another possible factor that may influence outcome is the time of diagnosis. Since symptoms may be ignored and obtaining medical history from a child is difficult or impossible, they may present late, while the detachment is old and involves the macula.

The relatively guarded prognosis of retinal detachment surgery in children was also noted in subgroups divided according to the aetiology. Better anatomical outcome was achieved in older children aged 15-19 years than in younger children up to 9 years old, probably because, for surgical purposes, older children resemble adults. ${ }^{19,31}$ In a previous study, we found that the occurrence of retinal detachment in children following closed and open globe injury was comparable and lower than would be expected according to the Ocular Trauma Score. ${ }^{32}$ This new index allows prediction of the visual outcome in a population with ocular trauma according to the initial visual acuity, type of injury, and associated findings. ${ }^{33}$ Each parameter had a prognostic value with certain weight (raw points) and the sum of the weights determined the expected last visual acuity. Retinal redetachment in our study was relatively high even in the adult group, because ocular trauma was frequent in this group.

The limitations of our study are that it was retrospective and some data may have been overlooked or not recorded. The surgical techniques, which may influence the outcomes, were different between the groups because of different aetiologies. In our study, we analyzed the data as two distinct but heterogeneous groups with separate aetiologies and with inherent differences between children that may have chronic unrecognized detachments and amblyopia, and adults. However, our study has a different perspective on retinal detachment from previous ones. The importance of this study is in evaluating the incidence of pathologies associated with retinal detachment in children and adults and comparing between them. These data may be used as references and may allow correlation between the surgical outcomes and the preoperative status.

The anatomical and functional outcomes of retinal detachment in children were less successful than in adults and the success of additional procedures to reattach the retina were also less successful than in adults. These were due to higher incidence of associated preoperative ocular pathologies, ocular syndromes, and trauma and a higher postoperative complication rate including possible development of irreversible amblyopia. Paediatric retinal detachment as a group 
should therefore be addressed differently from adult retinal detachment.

\section{Acknowledgements}

We thank Orly Yakir, MA, and Nurit Carmi, PhD, for the statistical analysis.

\section{References}

1 Winslow RL, Tasman W. Juvenile rhegmatogenous retinal detachment. Ophthalmology 1978; 85: 607-618.

2 Haimann MH, Burton TC, Brown CK. Epidemiology of retinal detachment. Arch Ophthalmol 1982; 100: 289-292.

3 Karel I, Michalickova M. Pars plana vitrectomy in paediatric population: indications and long term results. Eur J Ophthalmol 1999; 9: 231-237.

4 Hudson JR. Retinal detachments in children. Trans Ophthalmol Soc UK 1965; 85: 79-91.

5 Arensten JJ, Welch RB. Retinal detachment on the young individual: a survey of 100 cases seen at the Wilmer Institute. J Pediatr Ophthalmol 1965; 11: 198-202.

6 Hilton GF, Norton EWD. Juvenile retinal detachment. Mod Probl Ophthalmol 1969; 8: 325-341.

7 Tasman W. Retinal detachment in children. Trans Am Acad Ophthalmol Otolaryngol 1967; 71: 455-460.

8 Chisholm L. Retinal detachment in children. Can J Ophthalmol 1971; 6: 62-67.

9 Fivgas GD, Capone Jr A. Paediatric rhegmatogenous retinal detachment. Retina 2001; 21: 101-106.

10 Michels RG, Wilkinson CP, Rice JA. Retinal Detachment. St Luis: Mosby, 1990.

11 Tarkkanen A, Laatikainen L. Coats' disease: clinical, angiographic, histopathological findings and clinical management. Br J Ophthalmol 1983; 67: 766-776.

12 Schmidt-Erfurth U, Lucke K. Vitreoretinal surgery in advanced Coats' disease. Ger J Ophthalmol 1995; 4: 32-36.

13 Butler TKH, Kiel AW, Orr GM. Anatomical and visual outcome of retinal detachment surgery in children. $\mathrm{Br} \mathrm{J}$ Ophthalmol 2001; 85: 1437-1439.

14 Rostomian K, Thach AB, Isfahani A, Pakkar A, Pakkar R, Borchert M. Open globe injury in children. J AAPOS 1998; 2: 234-238.

15 Farr AK, Hairston RJ, Humayun MU, Marsh MJ, Pieramici DJ, MacCumber MW et al. Open globe injuries in children: a retrospective analysis. J Pediatr Ophthalmol Strabismus 2001; 38: 72-77.

16 Rosner M, Treister G, Belkin M. Epidemiology of retinal detachment in childhood and adolscence. J Pediatr Ophthalmol Strabismus 1987; 24: 42-44.
17 Hudson JR. The role of trauma in juvenile retinal detachment. Mod Probl Ophthalmol 1968; 8: 242-248.

18 Minoda K, Tanagami S, Takeuchi S, Kato A, Kawakami K. Juvenile retinal detachment. Jpm J Clin Ophthalmol 1978; 32: 59-67.

19 Scott JD. Congenital myopia and retinal detachment. Trans Ophthalmol Soc UK 1980; 100: 69-71.

20 Okinami S, Ogino N, Nishimura T, Tano Y. Juvenile retinal detachment. Ophthalmologica 1987; 194: 195-201.

21 Häring G, Wiechens B. Long-term results after scleral buckling surgery in uncomplicated juvenile retinal detachment without proliferative vitreoretinopathy. Retina 1998; 18: 501-505.

22 The Eye Disease Case-Control Study Group. Risk factors for idiopathic rhegmatogenous retinal detachment. Am J Epidemiol 1993; 137: 749-757.

23 Favre M, Goldmann H. Zur genese der hinterenglaskorperabhebung. Ophthalmologica 1956; 132: 86-97.

24 Foos RY. Posterior vitreous detachment. Tran Amer Acad Ophthalmol Otolaryngol 1972; 76: 480-497.

25 Bonnet $\mathrm{M}$. The development of severe proliferative vitreoretinopathy after retinal detachment surgery. Grade B: a determining risk factor. Graefe's Arch Clin Exp Ophthalmol 1988; 226: 201-205.

26 Heimann H, Bornfeld N, Friedrichs W, Helbig H, Kellner U, Korra A et al. Primary vitrectomy without scleral buckling for rhegmatogenous retinal detachment. Graefe's Arch Clin Exp Ophthalmol 1996; 234: 561-568.

27 Duquesne N, Bonnet M, Adeleine P. Preoperative vitreous hemorrhage associated with rhegmatogenous retinal detachment: a risk factor for postoperative retinal detachment. Graefe's Arch Clin Exp Ophthalmol 1996; 234: 677-692.

28 Hooymans JM, de Lavalette VW, Oey AG. Formation of proliferative vitreoretinopathy in primary rhegmatogenous retinal detachment. Doc Ophthalmol 2000; 100: 39-42.

29 La Heij EC, Derhaag PF, Hendrikse F. Results of scleral buckling operations in primary rhegmatogenous retinal detachment. Doc Ophthalmol 2000; 100: 17-25.

30 Akabane N, Yamamoto S, Tsukahara I, Ishida M, Mitamura Y, Yamamoto T. Surgical outcomes in juvenile retinal detachment. Jpn J Ophthalmol 2001; 45: 409-411.

31 Fenton PJ, Blach RK, Davis EWG, Bedford MA, Fison LG. Retinal detachment in children. Mod Probl Ophthalmol 1969; 8: 249-255.

32 Sarrazin L, Hemo I, Averbukh E, Halpert M, Rumelt S. Traumatic retinal detachment in the paediatric population- a comparison between open and closed globe injury. Am J Ophthalmol 2004; 137: 1042-1049.

33 Kuhn F, Maisiak R, Mann L, Morris R, Witherspoon CD. The OTS: predicting the final vision of the injured eye. In: Kuhn F, Pieramici DJ (eds.). Ocular Trauma. Principles and Practice. Thieme: New York, 2002, pp 9-13. 